\title{
NOTES ON A FAMILY OF INHOMOGENEOUS LINEAR ORDINARY DIFFERENTIAL EQUATIONS
}

\begin{abstract}
FENG QI
Institute of Mathematics, Henan Polytechnic University, Jiaozuo City, Henan Province, 454010, China; College of Mathematics, Inner Mongolia University for Nationalities, Tongliao City, Inner Mongolia Autonomous Region, 028043, China; Department of Mathematics, College of Science, Tianjin Polytechnic University, Tianjin City, 300387, China
\end{abstract}

\section{JING-LIN WANG}

Department of Mathematics, College of Science, Tianjin Polytechnic University, Tianjin City, 300387, China

\section{BAI-NI GUO}

School of Mathematics and Informatics, Henan Polytechnic University, Jiaozuo City, Henan Province, 454010, China

\begin{abstract}
In the note, the authors find several simple and explicit forms for a family of inhomogeneous linear ordinary differential equations studied in "D. Lim, Differential equations for Daehee polynomials and their applications, J. Nonlinear Sci. Appl. 10 (2017), no. 4, 1303-1315; Available online at http://dx.doi.org/10.22436/jnsa.010.04.02'.
\end{abstract}

\section{Motivation And main Results}

In [3, Theorem 1], it was obtained inductively and recurrently that the differential equations

$$
\frac{\partial^{n} F(t, x)}{\partial t^{n}}=\frac{1}{t^{n}}\left[\sum_{i=0}^{n}\left(a_{i}(n, x)+\frac{b_{i}(n, x)}{\ln (1+t)}\right)\left(\frac{t}{1+t}\right)^{i}\right] F(t, x), \quad n \geq 0
$$

E-mail addresses: qifeng618@gmail.com, qifeng618@hotmail.com, qifeng618@qq.com, jing-lin.wang@hotmail.com, bai.ni.guo@gmail.com, bai.ni.guo@hotmail.com 2010 Mathematics Subject Classification. 11B73, 11M35, 26A48, 26D07, 34A05, 44A10. Key words and phrases. simple form; explicit form; differential equation; Lerch transcendent; logarithmic function.

This paper was typeset using $\mathcal{A}_{\mathcal{M}} \mathcal{S}$-LATEX. 
have the same solution

$$
F(t, x)=\frac{\ln (1+t)}{t}(1+t)^{x}
$$

where $a_{0}(n, x)=(-1)^{n} n$ ! and $b_{0}(n, x)=0$ for all $n \geq 0$,

$$
\begin{aligned}
a_{j}(n, x)=(x)_{j}(-1)^{n-j}(n-j) ! & \sum_{i_{j-1}=0}^{n-j} \sum_{i_{j-2}=0}^{n-j-i_{j-1}} \\
& \cdots \sum_{i_{i}=0}^{n-j-i_{j-1}-\cdots-i_{2}}\left(n-i_{j-1}-\cdots-i_{1}-j+1\right)
\end{aligned}
$$

and

$$
\begin{aligned}
b_{j}(n, x)=\sum_{k=0}^{j-1} \prod_{i=0}^{j-1} \frac{(x-i)(-1)^{n-j}(n-j) !}{x-k} \sum_{i_{j-1}=0}^{n-j} \sum_{i_{j-2}=0}^{n-j-i_{i-1}} \\
\cdots \sum_{i_{1}=0}^{n-j-i_{j-1}-\cdots-i_{2}}{ }^{\left.n-i_{j-1}-\cdots-i_{1}-j+1\right)}\left(n-i_{j}\right.
\end{aligned}
$$

for $1 \leq j \leq n+1$, and $(x)_{n}=\prod_{k=0}^{n-1}(x-k)$ is the falling factorial.

It is clear that the above expressions for $a_{j}(n, x)$ and $b_{j}(n, x)$ are very difficult to compute by hand or by computer. The derivation of the quantities $a_{j}(n, x)$ and $b_{j}(n, x)$ in $[3]$ is much long and tedious.

The aim of this paper is to alternatively supply several new, simple, and explicit expressions for $a_{j}(n, x)$ and $b_{j}(n, x)$. In other words, the aim of this paper is to alternatively provide several new, simple, and explicit forms for the family of differential equations in (1.1).

Our main results can be stated as the following theorems.

Theorem 1. For $n \geq 0$, the function $F(t, x)$ defined in 1.2 satisfies

$$
\frac{\partial^{n} F}{\partial t^{n}}=(-1)^{n} \frac{n !}{t^{n}}\left\{\sum_{i=0}^{n}(-1)^{i} \frac{(x)_{i}}{i !}\left[1-\frac{1}{\ln (1+t)} \sum_{k=1}^{n-i} \frac{1}{k}\left(\frac{t}{1+t}\right)^{k}\right]\left(\frac{t}{1+t}\right)^{i}\right\} F
$$

and

$$
\frac{\partial^{n} F}{\partial t^{n}}=\frac{n !}{(1+t)^{n}}\left\{\sum_{i=0}^{n}(-1)^{i} \frac{(x)_{n-i}}{(n-i) !}\left[1-\frac{1}{\ln (1+t)} \sum_{k=1}^{i} \frac{1}{k}\left(\frac{t}{1+t}\right)^{k}\right]\left(\frac{1+t}{t}\right)^{i}\right\} F .
$$

Theorem 2. Let

$$
\Phi(z, s, a)=\sum_{k=0}^{\infty} \frac{z^{k}}{(a+k)^{s}}, \quad a \neq 0,-1, \ldots
$$

denote the Lerch transcendent. Then

$$
\frac{\partial^{n} F(t, x)}{\partial t^{n}}=\frac{(-1)^{n} n !}{(1+t)^{n-x+1}} \sum_{i=0}^{n}(-1)^{i} \frac{(x)_{i}}{i !} \Phi\left(\frac{t}{1+t}, 1, n-i+1\right)
$$

and

$$
\frac{\partial^{n} F(t, x)}{\partial t^{n}}=\frac{n !}{(1+t)^{n-x+1}} \sum_{i=0}^{n}(-1)^{i} \frac{(x)_{n-i}}{(n-i) !} \Phi\left(\frac{t}{1+t}, 1, i+1\right)
$$

for $n \geq 0$. 


\section{A LEMMA}

In order to prove our main results in Theorems 1 and 2, we need the following lemma.

Lemma 1 ([7, Theorem 2]). Let

$$
f(x)= \begin{cases}\frac{\ln x}{x-1}, & 0<x \neq 1 \\ 1, & x=1\end{cases}
$$

For $n \geq 0$, the $n$th derivative of $f(x)$ can be computed by

$$
f^{(n)}(x)= \begin{cases}\frac{(-1)^{n} n !}{(x-1)^{n+1}}\left[\ln x-\sum_{k=1}^{n} \frac{1}{k}\left(\frac{x-1}{x}\right)^{k}\right], & 0<x \neq 1 \\ (-1)^{n} \frac{n !}{n+1}, & x=1\end{cases}
$$

and

$$
f^{(n)}(x)= \begin{cases}(-1)^{n} \frac{n !}{x^{n+1}} \Phi\left(\frac{x-1}{x}, 1, n+1\right), & 0<x \neq 1 \\ (-1)^{n} \frac{n !}{n+1}, & x=1 .\end{cases}
$$

\section{Proofs of Theorems 1 and 2}

We now start out to prove our main results in Theorems 1 and 2

Proof of Theorem 1. By 2.1, it is straightforward that

$$
\begin{gathered}
\frac{\partial^{n} F(t, x)}{\partial t^{n}}=\sum_{i=0}^{n}\left(\begin{array}{c}
n \\
i
\end{array}\right)\left[\frac{\ln (1+t)}{t}\right]^{(n-i)}\left[(1+t)^{x}\right]^{(i)} \\
=\sum_{i=0}^{n}\left(\begin{array}{c}
n \\
i
\end{array}\right) \frac{(-1)^{n-i}(n-i) !}{t^{n-i+1}}\left[\ln (1+t)-\sum_{k=1}^{n-i} \frac{1}{k}\left(\frac{t}{1+t}\right)^{k}\right](x)_{i}(1+t)^{x-i} \\
=\frac{(1+t)^{x} \ln (1+t)}{t} \frac{(-1)^{n} n !}{t^{n}} \sum_{i=0}^{n} \frac{(-1)^{i}(x)_{i}}{i !}\left[1-\frac{1}{\ln (1+t)} \sum_{k=1}^{n-i} \frac{1}{k}\left(\frac{t}{1+t}\right)^{k}\right]\left(\frac{t}{1+t}\right)^{i} \\
=(-1)^{n} \frac{n !}{t^{n}}\left\{\sum_{i=0}^{n}(-1)^{i} \frac{(x)_{i}}{i !}\left[1-\frac{1}{\ln (1+t)} \sum_{k=1}^{n-i} \frac{1}{k}\left(\frac{t}{1+t}\right)^{k}\right]\left(\frac{t}{1+t}\right)^{i}\right\} F(t, x)
\end{gathered}
$$

and

$$
\begin{gathered}
\frac{\partial^{n} F(t, x)}{\partial t^{n}}=\sum_{i=0}^{n}\left(\begin{array}{c}
n \\
i
\end{array}\right)\left[\frac{\ln (1+t)}{t}\right]^{(i)}\left[(1+t)^{x}\right]^{(n-i)} \\
=\sum_{i=0}^{n}\left(\begin{array}{c}
n \\
i
\end{array}\right) \frac{(-1)^{i} i !}{t^{i+1}}\left[\ln (1+t)-\sum_{k=1}^{i} \frac{1}{k}\left(\frac{t}{1+t}\right)^{k}\right](x)_{n-i}(1+t)^{x-n+i} \\
=\frac{\ln (1+t)}{t(1+t)^{n-x}} \sum_{i=0}^{n}\left(\begin{array}{c}
n \\
i
\end{array}\right) \frac{(-1)^{i} i !}{t^{i}}\left[1-\frac{1}{\ln (1+t)} \sum_{k=1}^{i} \frac{1}{k}\left(\frac{t}{1+t}\right)^{k}\right](x)_{n-i}(1+t)^{i} \\
=\frac{F(t, x)}{(1+t)^{n}} \sum_{i=0}^{n}(-1)^{i} \frac{n !}{(n-i) !}\left[1-\frac{1}{\ln (1+t)} \sum_{k=1}^{i} \frac{1}{k}\left(\frac{t}{1+t}\right)^{k}\right](x)_{n-i}\left(\frac{1+t}{t}\right)^{i}
\end{gathered}
$$




$$
=\frac{n !}{(1+t)^{n}}\left\{\sum_{i=0}^{n}(-1)^{i} \frac{(x)_{n-i}}{(n-i) !}\left[1-\frac{1}{\ln (1+t)} \sum_{k=1}^{i} \frac{1}{k}\left(\frac{t}{1+t}\right)^{k}\right]\left(\frac{1+t}{t}\right)^{i}\right\} F(t, x) .
$$

The proof of Theorem 1 is complete.

Proof of Theorem 2. By (2.2), it is immediate that

$$
\begin{aligned}
\frac{\partial^{n} F(t, x)}{\partial t^{n}} & =\sum_{i=0}^{n}\left(\begin{array}{c}
n \\
i
\end{array}\right)\left[\frac{\ln (1+t)}{t}\right]^{(n-i)}\left[(1+t)^{x}\right]^{(i)} \\
& =\sum_{i=0}^{n}\left(\begin{array}{c}
n \\
i
\end{array}\right)(-1)^{n-i} \frac{(n-i) !}{(1+t)^{n-i+1}} \Phi\left(\frac{t}{1+t}, 1, n-i+1\right)(x)_{i}(1+t)^{x-i} \\
& =\frac{(-1)^{n} n !}{(1+t)^{n-x+1}} \sum_{i=0}^{n}(-1)^{i} \frac{(x)_{i}}{i !} \Phi\left(\frac{t}{1+t}, 1, n-i+1\right)
\end{aligned}
$$

and

$$
\begin{aligned}
\frac{\partial^{n} F(t, x)}{\partial t^{n}} & =\sum_{i=0}^{n}\left(\begin{array}{c}
n \\
i
\end{array}\right)\left[\frac{\ln (1+t)}{t}\right]^{(i)}\left[(1+t)^{x}\right]^{(n-i)} \\
& =\sum_{i=0}^{n}\left(\begin{array}{c}
n \\
i
\end{array}\right)(-1)^{i} \frac{i !}{(1+t)^{i+1}} \Phi\left(\frac{t}{1+t}, 1, i+1\right)(x)_{n-i}(1+t)^{x-n+i} \\
& =\frac{n !}{(1+t)^{n-x+1}} \sum_{i=0}^{n}(-1)^{i} \frac{(x)_{n-i}}{(n-i) !} \Phi\left(\frac{t}{1+t}, 1, i+1\right) .
\end{aligned}
$$

The proof of Theorem 2 is complete.

\section{REMARKS}

Finally we give several remarks about our main results mentioned and verified above.

Remark 1. Comparing (1.1) with (1.3) reveals that the formulas

$$
a_{i}(n, x)=(-1)^{n-i} \frac{n !}{i !}(x)_{i}
$$

and

$$
b_{i}(n, x)=-a_{i}(n, x) \sum_{k=1}^{n-i} \frac{1}{k}\left(\frac{t}{1+t}\right)^{k}=(-1)^{n-i+1} \frac{n !}{i !}(x)_{i} \sum_{k=1}^{n-i} \frac{1}{k}\left(\frac{t}{1+t}\right)^{k}
$$

should be valid for all $i, n \geq 0$.

Remark 2. The idea of this paper comes from the articles [1, 2, 4, 5, 6, 7, 8, 9, 10, 11, 12, 13, 14, and the closely related references therein.

\section{REFERENCES}

[1] B.-N. Guo and F. Qi, Explicit formulae for computing Euler polynomials in terms of Stirling numbers of the second kind, J. Comput. Appl. Math. 272 (2014), 251-257; Available online at http://dx.doi.org/10.1016/j.cam.2014.05.018

[2] B.-N. Guo and F. Qi, Some identities and an explicit formula for Bernoulli and Stirling numbers, J. Comput. Appl. Math. 255 (2014), 568-579; Available online at http://dx.doi. org/10.1016/j.cam.2013.06.020 
[3] D. Lim, Differential equations for Daehee polynomials and their applications, J. Nonlinear Sci. Appl. 10 (2017), no. 4, 1303-1315; Available online at http://dx.doi.org/10.22436/ jnsa.010.04.02

[4] F. Qi, Explicit formulas for the convolved Fibonacci numbers, ResearchGate Working Paper (2016), available online at http://dx.doi.org/10.13140/RG.2.2.36768.17927

[5] F. Qi and B.-N. Guo, Explicit formulas and nonlinear ODEs of generating functions for Eulerian polynomials, ResearchGate Working Paper (2016), available online at http://dx. doi.org/10.13140/RG.2.2.23503.69288

[6] F. Qi and B.-N. Guo, Identities of the Chebyshev polynomials, the inverse of a triangular matrix, and identities of the Catalan numbers, Preprints 2017, 2017030209, 21 pages; Available online at http://dx.doi.org/10.20944/preprints201703.0209.v1

[7] F. Qi and B.-N. Guo, Some properties of a solution to a family of inhomogeneous linear ordinary differential equations, Preprints 2016, 2016110146, 11 pages; Available online at http://dx.doi.org/10.20944/preprints201611.0146.v1

[8] F. Qi and B.-N. Guo, Some properties of the Hermite polynomials and their squares and generating functions, Preprints 2016, 2016110145, 14 pages; Available online at http://dx. doi.org/10.20944/preprints201611.0145.v1

[9] F. Qi and B.-N. Guo, Viewing some nonlinear ODEs and their solutions from the angle of derivative polynomials, ResearchGate Working Paper (2016), available online at http: //dx.doi.org/10.13140/RG.2.1.4593.1285

[10] F. Qi and B.-N. Guo, Viewing some ordinary differential equations from the angle of derivative polynomials, Preprints 2016, 2016100043, 12 pages; Available online at http: //dx.doi.org/10.20944/preprints201610.0043.v1

[11] F. Qi, J.-L. Wang, and B.-N. Guo, Simplifying two families of nonlinear ordinary differential equations, Preprints 2017, 2017030192, 6 pages; Available online at http://dx.doi.org/10. 20944/preprints201703.0192.v1

[12] F. Qi and J.-L. Zhao, Some properties of the Bernoulli numbers of the second kind and their generating function, J. Differ. Equ. Appl. (2017), in press. ResearchGate Working Paper (2017), available online at http://dx.doi.org/10.13140/RG.2.2.13058.27848

[13] F. Qi and J.-L. Zhao, The Bell polynomials and a sequence of polynomials applied to differential equations, Preprints 2016, 2016110147, 8 pages; Available online at http: //dx.doi.org/10.20944/preprints201611.0147.v1

[14] J.-L. Zhao, J.-L. Wang, and F. Qi, Derivative polynomials of a function related to the ApostolEuler and Frobenius-Euler numbers, J. Nonlinear Sci. Appl. 10 (2017), no. 4, 1345-1349; Available online at http://dx.doi.org/10.22436/jnsa.010.04.06

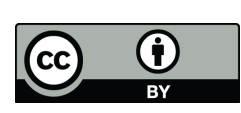

(C) 2017 by the authors; licensee Preprints, Basel, Switzerland. This article is an open access article distributed under the terms and conditions of the Creative Commons Attribution (CC BY) license http://creativecommons.org/licenses/by/4.0/).

$U R L:$ https://qifeng618.wordpress.com

$U R L:$ http://orcid.org/0000-0001-6725-533X

$U R L:$ http://www.researchgate.net/profile/Bai-Ni_Guo/ 